\title{
Lower limits on the maximum orbital frequency around rotating strange stars
}

\author{
D. Gondek-Rosińska ${ }^{1,2}$, N. Stergioulas ${ }^{3}$, T. Bulik ${ }^{2}$, W. Kluźniak ${ }^{4}$, and E. Gourgoulhon ${ }^{1}$ \\ 1 Département d'Astrophysique Relativiste et de Cosmologie UMR 8629 du CNRS, Observatoire de Paris, \\ 92195 Meudon Cedex, France \\ 2 Nicolaus Copernicus Astronomical Center, Bartycka 18, 00-716 Warszawa, Poland \\ 3 Department of Physics, Aristotle University of Thessaloniki, 54006 Thessaloniki, Greece \\ ${ }^{4}$ Institute of Astronomy, Zielona Góra University, ul. Lubuska 2, 65265 Zielona Góra, Poland
}

Received 7 September 2001 / Accepted 20 September 2001

\begin{abstract}
Observations of $\mathrm{kHz}$ quasi-periodic oscillations (QPOs) in the X-ray fluxes of low-mass X-ray binaries (LMXBs) have been used in attempts to constrain the external metric of the compact members of these binaries, as well as their masses and the equations of state of matter at supranuclear denisties. We compute the maximum orbital frequency of stable circular motion around uniformly rotating strange stars described by the MIT bag model. The calculations are performed for both normal and supramassive constant baryon mass sequences of strange stars rotating at all possible rates. We find the lower limits on the maximum orbital frequency and discuss them for a range of masses and for all rotational frequencies allowed in the model considered. We show that for slowly and moderately rotating strange stars the maximum value of orbital frequency can be a good indicator of the mass of the compact object. However, for rapidly rotating strange stars the same value of orbital frequency in the innermost stable circular orbit is obtained for stars with masses ranging from that of a planetoid to about three solar masses. At sufficiently high rotation rates of the strange star, the rotational period alone constrains the stellar mass to a surprisingly narrow range.
\end{abstract}

Key words. dense matter - equation of state - stars: neutron - stars: binaries: general - X- rays: stars

\section{Introduction}

The recently-discovered $\mathrm{kHz}$ quasi-periodic oscillations may be used as a probe of the inner regions of accretion disks in compact stars and hence also of some of the properties of the central object. For example, these oscillations have been used to derive estimates of the mass of the central source in some sources (e.g., Kaaret et al. 1997; Zhang et al. 1998; Kluźniak 1998). All these estimates assume that the maximum observed QPO frequency is the orbital frequency at the innermost stable circular orbit (ISCO), as suggested by Kluźniak et al. (1990).

In the first study of the possible presence of strange stars in those LMXBs which exhibit $\mathrm{kHz}$ QPOs, Bulik et al. (1999a,b) showed that slowly rotating strange stars, described by the simple MIT bag model with massless and non-interacting quarks, have orbital frequencies at the marginally stable orbit higher than the maximum frequency of $1.07 \mathrm{kHz}$ in $4 \mathrm{U} 1820-30$, reported by

Send offprint requests to: D. Gondek-Rosińska, e-mail: Dorota.Gondek@obspm.fr
Zhang et al. (1998). However, the ISCO frequencies can be as low as $1 \mathrm{kHz}$ when more sophisticated models of quark matter (MIT bag with massive strange quarks and lowest order QCD interactions) and/or rapid stellar rotation are taken into account (Stergioulas et al. 1999; Zdunik et al. 2000a,b). The main result of these considerations was that the lowest orbital frequency at the ISCO is attained either for non-rotating massive configurations close to their maximum mass limit, or for configurations at the Keplerian mass-shedding limit. In order to obtain an orbital frequency in the ISCO as low as $1.07 \mathrm{kHz}$ for slowly rotating strange stars one has to consider a specific set of (rather extreme) MIT bag model parameters (Zdunik et al. 2000a,b) allowing gravitational masses is higher than $2.2 M_{\odot}$. In contrast, for stars rotating close to the Keplerian limit, a low orbital frequency at the ISCO can be obtained for a broad range of stellar masses (Stergioulas et al. 1999).

A new description of quark matter (Dey et al. 1998) has been used to constructe numerical models of static strange stars and strange stars rotating with two different 
fixed frequencies, $200 \mathrm{~Hz}$ and $580 \mathrm{~Hz}$, and for calculating frequencies of marginally stable orbits around these stars (Datta et al. 2000). The authors conclude that very high QPO frequencies in the range of 1.9 to $3.1 \mathrm{kHz}$ would imply the existence of a non-magnetized strange star in an X-ray binary rather than a neutron star. Gondek-Rosińska et al. (2001) calculated the maximum orbital frequencies for the Dey et al. quark stars rotating at various rotation rates and showed that the rotational and maximum orbital frequencies for these stars are indeed much higher than those for neutron star models and for strange stars in the MIT bag model. The maximum orbital frequencies for the compact objects modeled with the Dey et al. (1998) equation of state are $\geq 1.5 \mathrm{kHz}$, and always higher than the $\mathrm{kHz}$ QPO frequencies observed to date.

Recently, new complications in the problem were pointed out. The innermost stable circular orbit exists also in Newtonian gravity (Kluźniak et al. 2000; Amsterdamski et al. 2001; Zdunik \& Gourgoulhon 2001). The presence of a crust has been investigated for normal evolutionary sequences by Zdunik et al. (2001). Typically the crust increases the maximum orbital frequency at the Keplerian limit.

In this paper we consider constant baryon mass sequences of strange stars described by the simplest MIT bag model (with massless and non-interacting particles) for various values of the baryon mass and calculate the frequency in the co-rotating ISCO (if it exists) for each sequence. We find and discuss lower limits to the maximum orbital frequency (taking into account various secular and dynamical stability limits, as well as physical constrains relevant for accretion in LMXBs). We then extend our results to all MIT equations of state. Our calculations are described in Sect. 2 and the results are presented in Sect. 3. Finally, Sect. 4 contains a summary of our results.

\section{The model star calculations}

Typically, strange stars are modeled (Alcock et al. 1986; Haensel et al. 1986) with an equation of state based on the MIT-bag model of quark matter, in which quark confinement is described by an energy term proportional to the volume (Farhi \& Jaffe 1984). The equation of state is given by the simple formula

$P=a\left(\rho-\rho_{0}\right) c^{2}$,

where $P$ is the pressure, $\rho$ the mass-energy density, and $c$ the speed of light. It was shown by Zdunik (2000) that all MIT bag models can be approximated very well by Eq. (1), where $a$ is a constant of model-dependent value in the range 0.289 (for a strange quark mass of $m_{\mathrm{s}}=250 \mathrm{MeV}$ ) to $1 / 3$ (for massless quarks). In the numerical calculations reported in the present paper we describe strange quark matter using the simplest MIT bag model (with massless and non-interacting quarks), accordingly we take $a=1 / 3$ and $\rho_{0}=4.2785 \times 10^{14} \mathrm{~g} \mathrm{~cm}^{-3} \equiv \rho_{\text {fid }}$ (corresponding to a bag constant of $B=60 \mathrm{MeV} \mathrm{fm}^{-3}$ ). In general, Eq. (1) corresponds to self-bound matter with density $\rho_{0}$ at zero

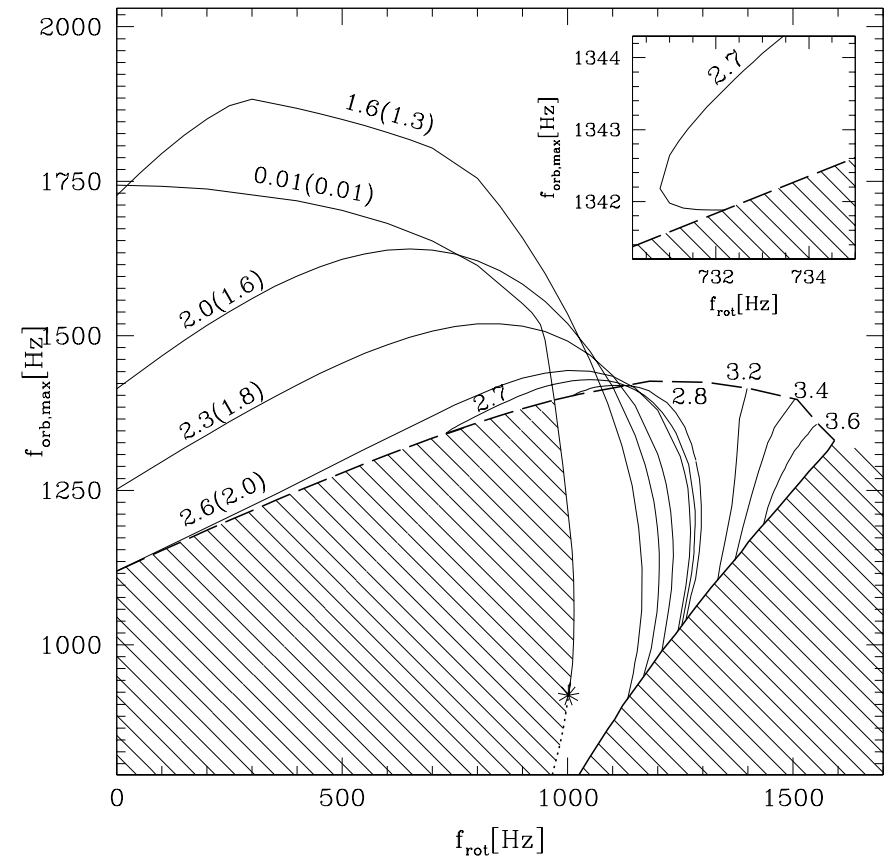

Fig. 1. The dependence of the maximum orbital frequency on the frequency of rotation $\Omega / 2 \pi$. Each thin solid line corresponds to a sequence of equilibrium models with a constant baryon mass, and is labeled by this baryon mass in solar mass units, as well as (in parentheses) by the gravitational mass of the static configuration, if it exists. The dashed line indicates stars marginally stable to axisymmetric perturbations. The angular momentum increases along each sequence from $J=0$ for static configurations, or $J_{\min }$ on the dashed line for supramassive stars, to $J_{\max }$ for the Keplerian configurations (thick solid line). One sequence for a very low mass star (with $M_{\mathrm{b}}=0.01 M_{\odot}$ ) is shown, the critical point on this sequence for Newtonian dynamical instability to non-axisymmetric perturbations is indicated by an asterisk and dynamically unstable configurations are denoted with the dotted line.The hatched area corresponds to a region excluded for rotating strange stars in the model considered. The inset shows in detail how the supramassive sequence reaches the stability limit to quasiradial oscillation.

pressure and with a fixed sound velocity $(\sqrt{a} c)$ at all pressures. For a fixed value of $a$, all stellar parameters are subject to scaling relations with appropriate powers of $\rho_{0}$ (see, e.g., Witten 1984; Zdunik 2000). Approximate scalings with $a$ were considered by Stergioulas et al. (1999) for the limiting case of rotation at Keplerian frequencies. Although a precise scaling-law with $a$ for other cases has not been derived, we will show that the lower limits on the maximum ISCO orbital frequency obtained for the simplest MIT bag model can easily be used to find lower limits on the maximum orbital frequency for any other value of $a$ and $\rho_{0}$.

We have calculated the maximum orbital frequency around uniformly rotating strange stars using two different highly accurate relativistic codes. The first code is based on the multi-domain spectral methods developed by Bonazzola et al. (1998). This method has been used previously for calculating properties of rapidly rotating strange 
stars described by the MIT bag model (Gourgoulhon et al. 1999) and by the Dey model (Gondek-Rosińska et al. 2000a). This code was also used to calculate the maximum orbital frequency around normal sequences of MIT strange stars (Zdunik et al. 2000b; Zdunik \& Gourgoulhon 2001) and of the Dey model strange stars (Gondek-Rosińska et al. 2001). The second code (Stergioulas \& Friedman 1995; see Stergioulas 1998 for a description) has been used to compute accurate constant baryon mass sequences and models at the Keplerian mass-shedding limit, where the first code has difficulties converging to a solution. In this code the equilibrium models are obtained following the KEH method (Komatsu et al. 1989), in which the field equations are converted to integral equations using appropriate Green's functions. Models computed by both codes agree very well in all computed properties.

We calculate the maximum frequency of a co-rotating test particle in a stable circular orbit in the equatorial plane about a strange star, $f_{\text {orb, max }}$. By testing stability of the orbital motion, we determine whether or not stable orbits extend to the surface of the star (at radius $R_{*}$ ). If they do, the maximum orbital frequency is the Keplerian frequency at the surface, $f_{\text {orb, } \max }=f_{\text {orb }}\left(R_{*}\right)$. If they do not, we find the radius of the innermost marginally stable orbit, $R_{\mathrm{ms}}$, and its frequency $f_{\mathrm{ms}}$ (see, e.g. Friedman et al. 1986). In this latter case, the orbital frequency in the ISCO is the maximum circular orbital frequency, $f_{\text {orb } \max }=f_{\mathrm{ms}}$. This frequency can then be compared with the observed $\mathrm{kHz}$ QPOs, having in mind some model of QPOs relating their frequency to the orbital frequency and the location of the ISCO (e.g., Kluźniak et al. 1990, Abramowicz \& Kluźniak 2001; Wagoner et al. 2001). In so doing, we neglect the effects of fluid pressure, magnetic fields, radiation drag, etc., on the location of the innermost circular orbit in the accretion disk.

We construct equilibrium sequences of rotating compact strange stars with constant baryon mass. We compute both normal and supramassive sequences. A sequence is called normal if it terminates at the zero angular momentum limit with a static, spherically symmetric solution, and it is called supramassive if it terminates at the axisymmetric stability limit, instead. The boundary between these two sequences is the sequence with the maximum baryon mass of a static configuration. The angular momentum (and the central density) of a star changes monotonically along each sequence.

For a supramassive constant baryon mass sequence a model is stable to axisymmetric perturbations, if $\left(\frac{\partial J}{\partial \rho_{\mathrm{c}}}\right)_{M_{\mathrm{b}}}<0\left(\right.$ or $\left.\left(\frac{\partial M}{\partial \rho_{\mathrm{c}}}\right)_{M_{\mathrm{b}}}<0\right)$ where $J$ is the angular momentum, $M$ is the gravitational mass, $M_{\mathrm{b}}$ is the baryon mass of the star and $\rho_{\mathrm{c}}$ is the central density of the star (Friedman et al. 1988; Cook et al. 1994).

The star in a sequence reaches the Keplerian limit (the mass-shedding limit) if the velocity at the equator of a rotating star is equal to the velocity of an orbiting particle at the surface of the star.

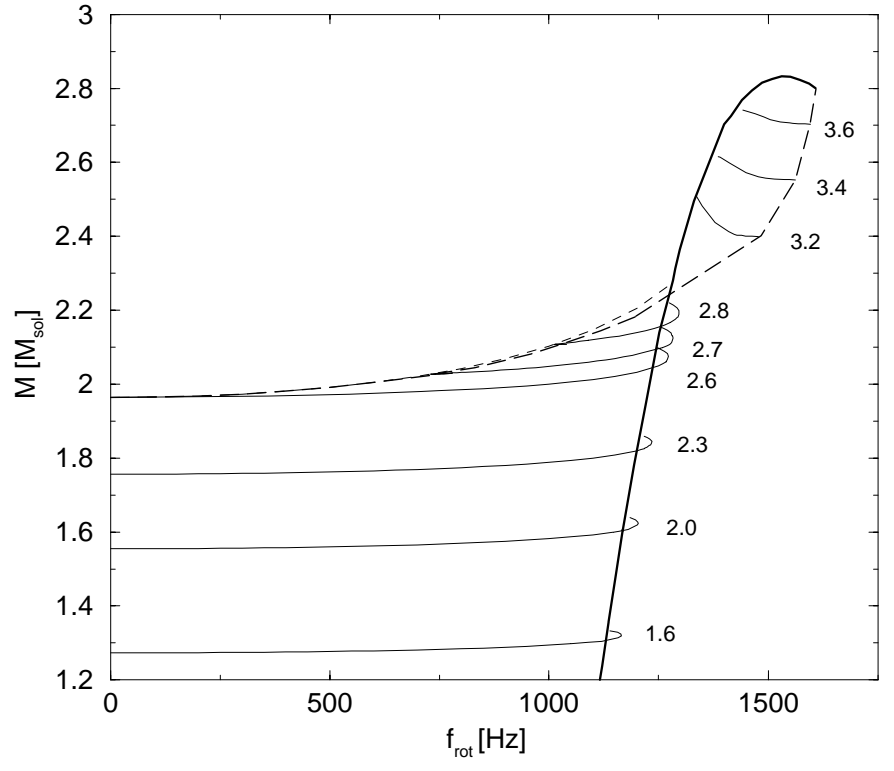

Fig. 2. Gravitational mass as a function of the rotational frequency for the same constant baryon mass sequences as shown in Fig. 1. The symbols are the same as in Fig. 1. The short dashed line corresponds to the upper limit on gravitational mass for the model considered.

\section{Results}

The properties of the ISCO around strange stars differ from those around neutron stars (Miller et al. 1998) - for strange (i.e., quark) stars of any mass the ISCO always exists at sufficiently rapid stellar rotation rate (Stergioulas et al. 1999; Zdunik et al. 2000b); and for strange stars of moderate and high baryon masses the ISCO always exists for any rotation rate (Zdunik et al. 2000b; GondekRosińska et al. 2001). For lower mass stars (e.g., the 1.6 $M_{\odot}$ baryon mass sequence in Fig. 1) stable orbits extend down to the stellar surface for moderate rotation rates, but the gap is present for both slowly and rapidly rotating stars. For lower masses yet, the gap is present only at high rotation rates and the ISCO is present even if relativistic effects are negligible (as in substellar-mass configurations, e.g., for $\left.M \leq 0.01 M_{\odot}\right)$. In the Newtonian limit, the gap between the marginally stable orbit and the stellar surface is produced by the oblateness of the rapidly rotating low-mass quark star (Kluźniak et al. 2000; Amsterdamski et al. 2001; Zdunik \& Gourgoulhon 2001). For Keplerian configurations the ISCO is always well above the stellar surface for all possible masses.

We present the dependence of the maximum orbital frequency on the frequency of rotation $f_{\text {rot }}=\Omega / 2 \pi$ for a set of constant baryon mass sequences of strange stars in Fig. 1. In Fig. 2 we show how the gravitational mass depends on rotational frequency for the considered sequences. The normal sequences start at $f_{\text {rot }}=0$, and continue with increasing angular momentum up to the Keplerian configuration. As it turns out, the very low mass limit, represented in Fig. 1 by the case of $M_{\mathrm{b}}=0.01 M_{\odot}$, is described well by a uniform-density 
fluid, i.e., the Maclaurin spheroid (Chandrasekhar 1969). For this sequence, an ISCO exists only for rotation rates larger than $\sim 970 \mathrm{~Hz}$, and we indicate the onset of the (Newtonian) dynamical instability to non-axisymmetric perturbations by an asterisk.

The intermediate-mass case is shown by the constant baryon mass sequence with the baryon mass $M_{\mathrm{b}}=$ 1.6 $M_{\odot}$, for which the ISCO does not exist between the rotation rates with frequency of $f_{\text {rot }} \approx 300 \mathrm{~Hz}$ and $f_{\text {rot }} \approx 800 \mathrm{~Hz}$ (see also Zdunik et al. 2000b).

More massive stars in normal sequences are shown for the cases of $M_{\mathrm{b}}=2.0 M_{\odot}$ and $2.3 M_{\odot}$. In these cases, the marginally stable orbit exists along the entire constant baryon mass sequence. The ISCO frequency initially increases with rotational frequency, as calculated in the slow-rotation approximation by Kluźniak \& Wagoner (1985). When the small-rotation-rate assumption is no longer satisfied, the ISCO frequency reaches a maximum and begins to decrease mainly due to the rotational deformation of the star (Sibgatulin \& Sunyaev 1998; Shibata \& Sasaki 1999). The sequence terminates when the star has reached the Keplerian configuration (mass-shedding limit). An extreme case of normal sequences is the sequence starting with the maximum mass static configuration of $M_{\mathrm{b}}=2.624 M_{\odot}$ (indicated as 2.6 $M_{\odot}$ in Figs. 1 and 2).

Supramassive sequences start at the onset of the instability to axisymmetric perturbations (thick dashed line in Fig. 1 and in subsequent figures). Sequences with $M_{\mathrm{b}}=$ $2.7 M_{\odot}$ and $2.8 M_{\odot}$ are examples of low mass supramassive sequences. The behavior of the maximum orbital frequency vs. frequency of rotation is basically similar to the normal sequences described above.

Finally, we present the sequences of models with $M_{\mathrm{b}}=$ 3.2, 3.4, and 3.6 $M_{\odot}$. For these models of extremely high mass, the rotational frequency decreases with increasing angular momentum because the stars are initially highly deformed. The ISCO frequency only decreases along these sequences until the stars reach the mass shedding limit. The configuration with the highest rotation frequency is not Keplerian. For a high-mass supramassive strange star sequence (e.g., $M_{\mathrm{b}}=3.2$ ), the Keplerian configuration is the one with the lowest rotational frequency in the sequence (see Fig. 2).

Note that the rotational frequency does not necessarily change monotonically along a constant baryon mass sequence, since a star changes its shape and, consequently, its moment of inertia with increasing angular momentum (see also Cook et al. 1992; Zdunik et al. 2000b; GondekRosińska et al. 2000a). For a lower-mass supramassive sequence and a normal sequence the rotational frequency decreases with increasing angular momentum just below the mass-shedding limit (characteristic turning back in Figs. 1 and 2 for each sequence). A similar behavior is observed in the case of models with small mass, e.g. for the constant baryon mass sequence with $M_{\mathrm{b}}=0.01 M_{\odot}$. Thus, the effect of spin-down with increasing angular momentum is of

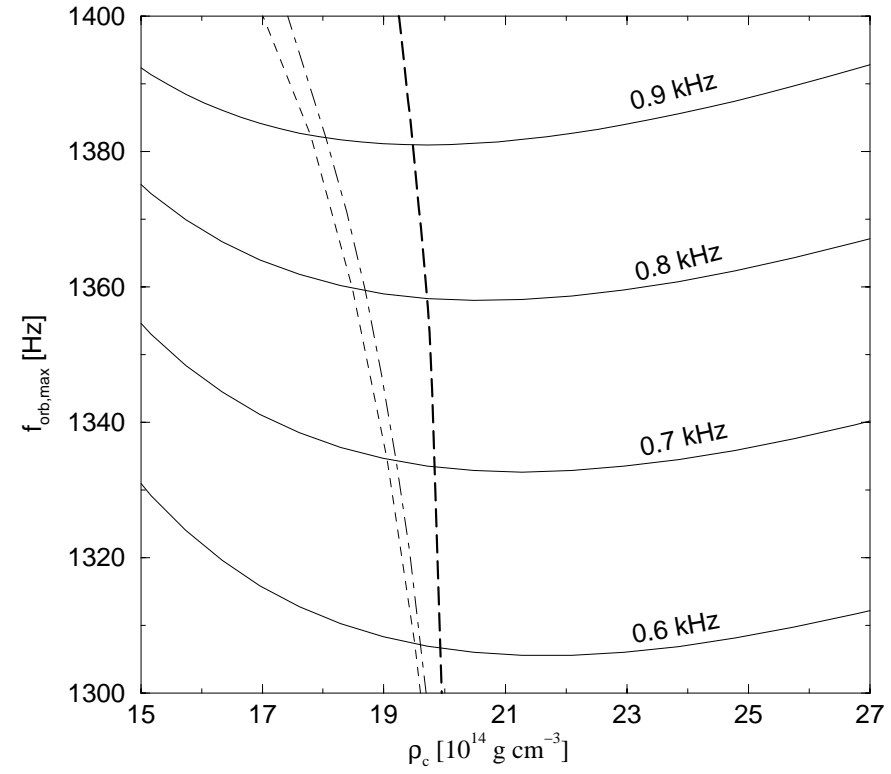

Fig. 3. The ISCO frequency as a function of central density for sequences with a fixed rotational frequency, as labeled. The short dashed line denotes the configurations with the maximum possible gravitational mass for given frequency (see Fig. 2), the dash-dotted line corresponds to stars with maximum baryon mass, and the thick dashed line represents the axisymmetric stability limit.

Newtonian origin, and is related to the change of shape of the star.

In Fig. 1 one can see that the value of the ISCO frequency alone is not a sufficient indicator for the mass of the compact object. For a rapidly rotating strange star the same value of orbital frequency at the innermost stable circular orbit, e.g. $1.2 \mathrm{kHz}$, can correspond to a range of stars differing in mass by a factor of a hundred, or even more, as stressed already by Kluźniak et al. (2000). However, it is worth noting that for rotational frequencies exceeding about $1.3 \mathrm{kHz}$, i.e., for rapidly rotating supramassive stars, the rotational period alone constrains the stellar mass to a narrow range.

\subsection{Lower limits on the ISCO frequency}

Figure 1 shows that at any given stellar rotational frequency the maximum orbital frequency $f_{\text {orb, max }}(f)$ is limited from below by the least frequency among the following three "extreme" sequences of stars: a) the marginally stable configuration to axisymmetric perturbations (thick dashed line); b) configurations rotating at Keplerian frequencies (thick solid line) and c) the Newtonian limit of low-mass stars, tending to Maclaurin spheroids when $M \rightarrow 0, J \rightarrow 0, \rho \rightarrow \rho_{0}$ (solid line ending with the asterisk). For all these limiting cases, an ISCO exists. The maximum orbital frequencies are also bounded from above by a fourth limiting line (not shown), which will be discussed elsewhere: the envelope of highest values of $f_{\text {orb,max }}$.

a) We find that at a given low or moderate value of stellar rotational frequency the lowest ISCO frequency 
is obtained for the configurations at the axisymmetric instability limit. As was shown by Gourgoulhon et al. (1999) and Gondek-Rosińska et al. (2000a) a supramassive strange star, in the same way as a supramassive neutron star (Cook et al. 1992), prior to its collapse to a black hole increases its spin as it loses angular momentum. In such cases, two models with the same baryon mass but with different central density and angular momentum can have the same rotational frequency (see inset in Fig. 1). Along a sequence of fixed rotational frequency, the model with the lowest ISCO frequency is not the model with the highest gravitational or highest baryon mass. The dependence of the ISCO frequency as function of the stellar central density for sequences of stars with a fixed rotational frequency is shown in Fig. 3. As the central density increases, the ISCO frequency decreases and the sequence passes through the configuration with maximum gravitational mass (denoted with short dashed line in Figs. 2 and 3 ), and then the maximum baryon mass configuration (denoted with the dash-dotted line). Before reaching the minimal value of $f_{\text {orb, } \max }$, the sequence reaches the limit of stability to axisymmetric perturbations (thick dashed line). Thus, for a fixed rotational frequency, the lowest possible maximum orbital frequency is attained for the model which is marginally stable to gravitational collapse.

b) At high rotation rates the lowest ISCO frequency is attained for the Keplerian (mass-shedding) configurations (thick solid line in Fig. 1). The stellar configurations at this limit are highly deformed. We calculated Keplerian configurations beginning with the fastest rotating stable configurations (with $M_{\mathrm{b}}=3.76 M_{\odot}$ ), ending with a strange star with $M_{\mathrm{b}}=0.4 M_{\odot}$. Note that for rapidly rotating (close to the Keplerian limit) strange stars, the ISCO frequency is smaller than the rotational frequency.

c) In the small-mass limit the ISCO only exists at high stellar rotation rates. This was discovered numerically, and the Newtonian origin of the ISCO in this case was first understood by comparison with toy models of disklike distributions of matter (Kluźniak et al. 2000; Zdunik \& Gourgoulhon 2001). In the Newtonian limit $(M \rightarrow 0$, $\left.J / M^{2} \rightarrow \infty\right)$ strange stars tend to a nearly uniformdensity configuration $\left(\rho \rightarrow \rho_{0}\right)$, and these can be described well by the Maclaurin spheroids, for which analytic formulae for orbital frequencies have been derived and found to agree with the numerical models (Amsterdamski et al. 2001). The functional dependence of the maximum orbital frequency on the stellar rotation rate is nearly universal for all strange stars with masses lower than $0.1 M_{\odot}$, in the model considered here.

\subsection{The lower limits on ISCO for other MIT bag models}

Figure 1 and the discussion above assumed the simplified MIT bag model with massless and non-interacting quarks. The results can easily be scaled for any value of the density at zero pressure, with the use of the scaling $f \propto \rho_{0}^{1 / 2}$,

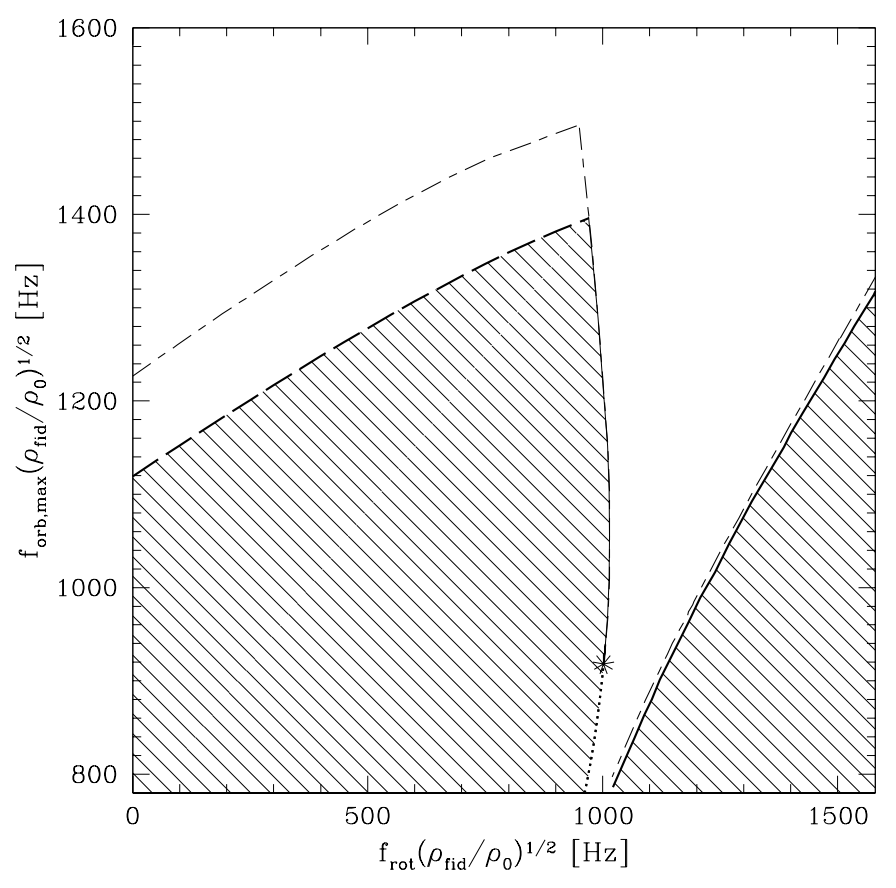

Fig. 4. The effect of non-zero strange quark mass on the lower limits on the maximum orbital frequency. The dependence on $\rho_{0}$ has been scaled out. Here, the thin dotted-dashed lines correspond to the limits for the case of $m_{\mathrm{s}}=250 \mathrm{MeV}$ $(a=0.289)$, and all other lines and symbols have the same meaning as in Fig. 1, which was obtained for $a=1 / 3\left(m_{\mathrm{s}}=0\right)$.

where $f$ denotes either of the frequencies (rotational or orbital), and $M \propto \rho_{0}^{-1 / 2}$. We can easily find lower limits on ISCO for any other MIT bag model since Zdunik (2000) showed that all MIT bag model equations of state can be well approximated by Eq. (1).

In order to find the dependence of the lower limits on $a$ we display in Fig. 4 the limits calculated for $m_{\mathrm{s}}=0$ and for $m_{\mathrm{s}}=250 \mathrm{MeV}$. We have chosen these values of the strange quark mass because it corresponds to the highest value $a=0.333$, and the lowest value of $a=0.289$ for the MIT bag model equation of state. The dependence on the density of quark matter at zero pressure has been scaled out by multiplying the frequencies with the square root of $\rho_{\text {fid }} / \rho_{0}$, where our fiducial value of $\rho_{0}$ was used: $\rho_{\text {fid }}=4.2785 \times 10^{14} \mathrm{~g} \mathrm{~cm}^{-3}$. The numerical values of the frequencies in Fig. 4 correspond to the value of the bag constant $B=60 \mathrm{MeV} \mathrm{fm}^{-3}$ used throughout this paper.

The limit on the maximum orbital frequency, set by the low mass stars, does not depend on the value of $a$. The limit at rapid rotation rates depends only slightly on the value of $a$. The main difference appears in the slow and moderate rotation regions, where the lower limit is set by the sequence of marginally stable stars. However, here one can use the fact that the limit is only shifted along the vertical axis. We find that the formula $f_{\text {orb,max }}\left(a, f_{\text {rot }}\right)=0.3 f_{\text {rot }}+f_{\text {orb, } \max }(a, 0)$ is a good approximation for describing the lower limit on the ISCO frequency for slowly and moderately rotating strange stars described by any MIT equation of state. Here, the 


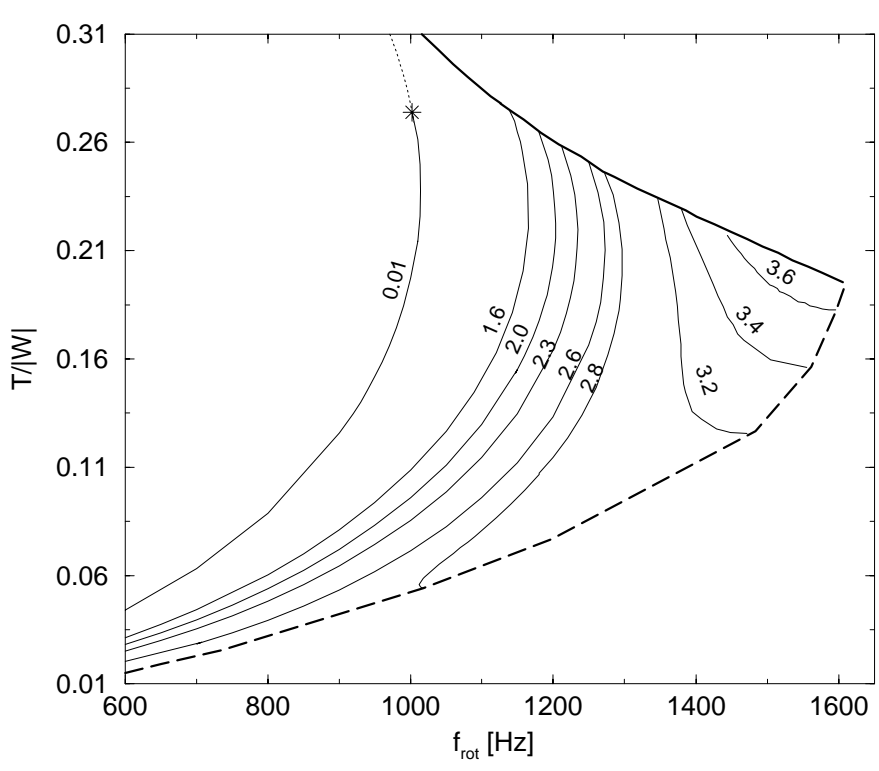

Fig. 5. The ratio of the kinetic energy to the absolute value of the gravitational potential energy (defined as in Friedman et al. 1986) as a function of the rotational frequency for several constant baryon mass sequences of MIT bag model strange stars. The meaning of the lines is the same as in Fig. 1. The critical value of $T /|W|$ for the onset of dynamical stability in the low mass limit is the Newtonian value of 0.27 indicated by an asterisk.

maximum orbital frequency of the nonrotating model is given by its Schwarzschild-metric value

$$
f_{\text {orb }, \max }(a, 0)=2.2 \mathrm{kHz} /\left(M(a) / M_{\odot}\right)
$$

(e.g., Kluźniak et al. 1990), and $M(a)$ is the maximum mass in general relativity of a static configuration of the (quark) fluid described by Eq. (1) - in short, the maximum mass of a non-rotating strange star.

\subsection{Factors that could alter the lower limits}

There are several factors which could affect the lower limits considered in the previous section. We discuss here: i) different kinds of instabilities that could operate in rapidly rotating strange stars, ii) the existence of a solid crust on the accreting strange stars, iii) details of the accretioninduced spin-up of strange stars in LMXBs.

i) Rapidly rotating compact stars may be subject to non-axisymmetric rotational instabilities. A widely used indicator for the onset of instability is the ratio of the kinetic energy to the absolute value of the gravitational potential energy $T /|W|$. We plot the value of $T /|W|$ (defined as in Friedman et al. 1986) for several constant baryon mass sequences in Fig. 5. The values of $T /|W|$ for strange stars rotating at the Keplerian velocity are very large and can be even higher than $T /|W|=0.32^{1}$. The $T /|W|$ is

\footnotetext{
${ }^{1}$ In Gourgoulhon et al. 1999 (see their Fig. 7), $T /|W|<$ 0.24 , because configurations denoted as Keplerian, were in fact configurations at which the numerical procedure stopped converging.
}

very high for all linear self-bound equations of state (see Gondek-Rosińska et al. 2000b for different MIT bag models and Gondek-Rosińska et al. 2000a for the strange stars described by the Dey model). The values of $T /|W|$ for strange stars are much larger than for any neutron star models (for which the maximum $T /|W|$ ranges typically from 0.1 to 0.14 , (see e.g. Nozawa et al. 1998). In contrast to neutron stars, the $T /|W|$ for strange stars increases with decreasing stellar mass for all models. Very large values of $T /|W|$ are characteristic not only for supramassive stars but also for rapidly rotating strange stars with small and moderate masses. The large value of $T /|W|$ results from a flat density profile combined with strong equatorial flattening of rapidly rotating strange stars. No stability analysis in general relativity has been performed yet for strange stars.

There exists two different classes and corresponding time-scales for non-axisymmetric instabilities: a dynamical instability, growing on the hydrodynamical time-scale, and secular instabilities (due to dissipative mechanisms) which grow on much longer time-scales. In Newtonian theory, rapidly rotating Maclaurin spheroids are secularly unstable to a bar-mode formation, if $T /|W|>(T /|W|)_{\text {crit }}=$ 0.1375 (Jacobi/Dedekind bifurcation point) and dynamically unstable if $(T /|W|>0.2738)$. A secular instability can grow only in the presence of dissipative mechanisms, such as shear viscosity (Roberts \& Stewartson 1963) or gravitational radiation (the ChandrasekharFriedman-Schutz instability (hereafter CFS instability; Chandrasekhar 1970; Friedman \& Schutz 1978; Friedman 1978). If both viscosity and gravitational radiation are operative, they act against each other. Which of the two dissipative mechanisms dominates is highly dependent on the temperature of the star. By a suitable choice of the strength of viscosity relative to gravitational radiation, it is possible to stabilize the Maclaurin sequence up to the point of dynamical instability (Shapiro \& Teukolsky 1983).

In general, the viscosity-driven instability could be operating in accreting (old and cold) compact objects, while the CFS instability could operate in both newly-born compact stars and in LMXBs. The most important pulsation modes for the CFS instability are the $r$ - and $f$-modes (see Stergioulas 1998; Andersson \& Kokkotas 2001; Friedman \& Lockitch 2001, for recent reviews of these instabilities).

The dynamical instability against bar-mode deformation of rapidly rotating polytropic stars was studied by Shibata et al. (2000) in full general relativity and by Saijo et al. (2001) in the first post-Newtonian approximation. The authors considered differentionally rotating proto-neutron stars. Their main result is that in general relativity the onset of dynamical instability occurs for a somewhat smaller critical value of $(T /|W|)_{\text {crit }} \sim$ $0.24-0.25$ than the Newtonian value $\sim 0.27$ for incompressible Maclaurin spheroids.

The few numerical studies of secular instabilities in rapidly rotating stars in general relativity show that nonaxisymmetric modes driven unstable by viscosity no longer 
coincide with those driven unstable by gravitational radiation. The $(T /|W|)_{\text {crit }}$ depends not only on the dissipative mechanism but also on the compaction parameter $M / R$ of a star. The viscosity-driven instability for neutron stars has been studied in general relativity by Bonazzola et al. (1996, 1998), who find that it sets in for $T /|W|$ somewhat larger than the Newtonian value of $(T /|W|)_{\text {crit }}=0.1375$. A stronger weakening of the viscosity-driven instability by general relativity is suggested by a post-Newtonian study of the viscosity-driven instability in incompressible stars by Shapiro \& Zane (1998), who find that the $(T /|W|)_{\text {crit }}$ could be as large as 0.25 for $M / R \sim 0.2$. On the other hand, the gravitational-radiation driven instability is strengthened by relativity and the bar $(l=2) f$ mode can become unstable for typical neutron star equations of state (Stergioulas \& Friedman 1998). For a wide range of realistic equations of state, Morsink et al. (1999) find that the critical $T /|W|$ for the onset of the instability in the $l=2 f$-mode is only $T /|W| \sim 0.08$ for models with mass $1.4 M_{\odot}$ and $T /|W| \sim 0.06$ for maximum mass models.

The CFS-instability of $r$-modes in strange stars has been studied by Madsen (2000), in the Newtonian limit. According to this study, the instability can operate at a minimum rotational frequency of $\sim 400 \mathrm{~Hz}$ and at a temperature of $T \sim 10^{7} \mathrm{~K}$. Since strange stars can, at most, have only a thin solid crust, they cannot store heat in the interior during accretion. Thus, accreting strange stars are likely to have interior temperatures comparable to $T \sim 10^{7} \mathrm{~K}$. If the $r$-mode instability is not damped by other mechanisms then the above results seem to rule out the presence of low and moderate mass stars with a normal quark matter in LMXBs (if the observed $1.07 \mathrm{kHz}$ QPO is the orbital frequency at the ISCO).

If, contrary to our current understanding, the $r$-mode instability is not effective in accreting strange stars, then the $f$-mode instability or the viscosity-driven instability could be more relevant. More detailed studies of these instabilities specifically for relativistic strange stars are needed. The dynamical instability, even if present at $T /|W| \sim 0.24$, only marginally affects the limits presented in Fig. 1.

ii) Another factor affecting the lower limits presented in Fig. 1 would be the presence of a solid crust. This effect has been discussed by Zdunik et al. (2001). The presence of the crust will not affect the lower limit in the small and moderate rotation case. Here the stability of the strange stars is basically determined by the stability of the strange core and the properties of the crust are negligible. However, for rapid rotation, when the lower limit on the maximum orbital frequency is determined by the Keplerian configurations, the presence of the crust will increase the lower limit. The crust is of low density and for rapidly rotating strange stars it becomes extended in the equatorial region. Thus the mass-shedding limit is reached at lower rotation rates than in the case of bare strange stars. Zdunik et al. (2001) showed that the values of maximum orbital frequency for strange stars with maximum crust (up to the neutron drip point), rotating at the massshedding limit, are about $20 \%$ larger compared to case of bare strange stars. Also, for strange stars with a crust, the ISCO exists only for models with large mass.

iii) When a strange star is spun-up by accretion of material that leaves the ISCO and plunges onto the surface of the star, the largest attainable rotation rate is not precisely the rotation rate at the mass-shedding limit. Material that leaves from the ISCO arrives at the surface of a strange star after following a path in which it conserves angular momentum. At the surface, the material has angular velocity larger than at the ISCO, but still somewhat smaller than the angular velocity needed to spin-up the star to the angular velocity of the equilibrium configuration at the mass-shedding limit. The difference between the maximum rotation and mass-shedding configurations is, however, small.

\section{Summary}

Relativistic orbital frequencies are crucially related to the spin-up of neutron stars and black holes and to high energy accretion phenomena in low mass X-ray binaries, including quasi-periodic oscillations in the X-ray flux. We compute the maximum orbital frequency of stable circular motion around strange stars, and present it as a function of the stellar rotation rate for normal and supramassive constant baryon mass sequences of stars rotating at all possible rates. We find that in contrast to neutron stars, the mass of a rapidly rotating strange star cannot be even approximately inferred from the orbital frequencies alone. For strange stars described by the MIT bag model, the same frequency in the innermost stable orbit (e.g., $1.2 \mathrm{kHz}$ ) is obtained for strange stars with rotational periods ranging from infinity to about $0.6 \mathrm{~ms}$, and the mass ranging from that of a planetoid to about three solar masses. One should note however that the rapidly rotating stars in this region are quite likely to be unstable.

We find that for slow to moderate rotation rates the lower limit on the maximum orbital frequency around strange stars is attained for configurations marginally stable to axisymmetric perturbations, while at rapid rotation rates it is reached for configurations at the mass-shedding limit. There are several factors which could affect the lower limit for rapidly rotating strange stars, such as different instabilities or the existence of a solid crust. The $r$-mode instability has the potential to rule out strange stars with small and moderate masses in LMXBs (assuming that the observed $1.07 \mathrm{kHz}$ QPO in $4 \mathrm{U} 1820-30$ is close to the Keplerian frequency at the innermost stable orbit of an accretion disk around a strange star). If the $r$-mode instability is not effective, then the $f$-mode instability and the viscosity-driven instability may become relevant. On the other hand, the dynamical instability only marginally affects our computed lower limits on the maximum orbital frequency. Our results apply to all MIT bag models. 
Acknowledgements. This work has been funded by the following grants: KBN grants 5P03D01721, 2P03D00418, 2P03D01816; the Greek-Polish Joint Research and Technology Programme EPAN-M.43/2013555 and the EU Programme "Improving the Human Research Potential and the SocioEconomic Knowledge Base" (Research Training Network Contract HPRN-CT-2000-00137).

\section{References}

Abramowicz, M., \& Kluźniak, W. 2001, A\&A, 374, L19

Alcock, C., Farhi, E., \& Olinto, A. 1986, ApJ, 310, 261

Amsterdamski, P., Bulik, T., Gondek-Rosińska, D., \& Kluźniak, W. 2001, A\&AL, in press [astro-ph/0012547]

Andersson, N., \& Kokkotas, K. D. 2001, Int. J. Mod. Phys. D, in press [gr-qc/0010102]

Bombaci, I., \& Datta, B. 2000, ApJ, 530, L69

Bonazzola, S., Frieben, J., \& Gourgoulhon, E. 1996, ApJ, 460, 379

Bonazzola, S., Frieben, J., \& Gourgoulhon, E. 1998b, A\&A, 331,280

Bonazzola, S., Gourgoulhon, E., \& Marck, J. A. 1998a, Phys. Rev. D., 58, 104020

Bulik, T., Gondek-Rosińska, D., \& Kluźniak, W. 1999a, A\&A, 344, L71

Bulik, T., Gondek-Rosińska, D., \& Kluźniak, W. 1999b, ApJ Letters and Communications, 38, 77

Chandrasekhar, S. 1969, Elipsoidal figures of equilibrium (Yale University Press, New Haven)

Chandrasekhar, S. 1970, Phys. Rev. Lett., 24, 611

Cook, G. B., Shapiro, S. L., \& Teukolsky, S. A. 1992, ApJ, 398, 203

Cook, G. B., Shapiro, S. L., \& Teukolsky, S. A. 1994, ApJ, 424, 823

Datta, B., Thampan, A. V., \& Bombaci, I. 2000, 355, L19

Dey, M., Bombaci, I., Dey, J., Ray, S., \& Samanta, B. C. 1998, Phys. Lett. B, 438, 123

Farhi, E., \& Jaffe, R. L. 1984, Phys. Rev. D, 30, 2379

Friedman, J. L. 1978, Commun. Math. Phys., 62, 247

Friedman, J. L., Ipser, J. R., \& Parker, L. R. 1986, ApJ, 304, 115; erratum published in ApJ, 351, 705 (1990)

Friedman, J. L., Ipser, J. R., \& Sorkin, R. D. 1988, ApJ, 325, 722

Friedman, J. L., \& Lockitch, K. H. 2001 [gr-qc/0102114]

Friedman, J. L., \& Shutz, B. F. 1978, ApJ, 222, 281

Gondek-Rosińska, D., Bulik, T., Kluźniak, W., Zdunik, J. L., \& Gourgoulhon, E. 2001, Proc. of the 4th Integral Meeting Alicante, in press [astro-ph/0012540]

Gondek-Rosińska, D., Bulik, T., Zdunik, J. L., et al. 2000a, A\&A, 363, 1005
Gondek-Rosińska, D., Haensel, P., Zdunik, J. L., \& Gourgoulhon, E. 2000b, ASP Conf. Ser., 202, 661 [astro-ph/0009282]

Gourgoulhon, E., Haensel, P., Livine, R., et al. 1999, A\&A, 349,851

Haensel, P., Zdunik, J. L., \& Schaeffer, R. 1986, A\&A, 160, 121

Kaaret, P., Ford, E. C., \& Chen, K. 1997, ApJ, 480, 127

Komatsu, H., Eriguchi, Y., \& Hachisu, I. 1989, MNRAS, 237, 355

Kluźniak, W. 1998, ApJ, 509, L37

Kluźniak, W., Bulik, T., \& Gondek-Rosińska, D. 2000, Proc. of the 4th Integral Meeting, in press [astro-ph/0011517]

Kluźniak, W., Michelson, P., \& Wagoner, R. V. 1990, ApJ, 358, 538

Kluźniak, W., \& Wagoner, R. V. 1985, ApJ, 297, 548

Madsen, J. 2000, Phys. Rev. Lett., 85, 10

Miller, M. C., Lamb, F. K., \& Cook, G. B. 1998, ApJ, 509, 793

Morsink, S. M., Stergioulas, N., \& Blattnig, S. R. 1999, ApJ, 510,854

Nozawa, T., Stergioulas, N., Gourgoulhon, E., \& Eriguchi, Y. 1998, A\&AS, 132, 431

Roberts, P. H., \& Stewartson, K. 1963, ApJ, 137, 777

Saijo, M., Shibata, M., Baumgarte, T. W., \& Shapiro, L. S. 2001, ApJ, 548, 919

Shapiro, S. L., \& Teukolsky, S. A. 1983, Black holes, white dwarfs, and neutron stars (New York: Wiley)

Shapiro, S. L., \& Zane, S. 1998, ApJS, 117, 531

Shibata, M., Baumgarte, T. W., \& Shapiro, S. L. 2000, ApJ, 542,453

Shibata, M., \& Sasaki, M. 1999, Phys. Rev. D, 60, 084002

Sibgatulin, N. R., \& Sunyaev, R. A. 1998, Astron. Lett. 24, 774

Stergioulas, N. 1998, Living Rev. Relativity, 1, 8, http:///www.livingreviews.org/Articles/Volume1/ 1998-8stergio/

Stergioulas, N., \& Friedman, J. L. 1995, ApJ, 444, 306

Stergioulas, N., Kluźniak, W., \& Bulik, T. 1999, A\&A, 352, L116

Wagoner, R. V., Silbergleit, A. S., \& Ortega-Rodríguez, M. 2001, ApJ, 559, L25

Witten, E. 1984, Phys. Rev., 30, 272

Zdunik, J. L. 2000, A\&A, 359, 311

Zdunik, J. L., Bulik, T., Kluźniak, W., Haensel, P., \& Gondek-Rosińska, D. 2000a, A\&A, 359, 143

Zdunik, J. L., \& Gourgoulhon, E. 2001, Phys. Rev. D63, 087501

Zdunik, J. L., Haensel, P., Gondek-Rosińska, D., \& Gourgoulhon, E. 2000b, A\&A 356, 612

Zdunik, J. L., Haensel, P., \& Gourgoulhon, E. 2001, A\&A, 372, 535

Zhang, W., Strohmayer, T. E., \& Swank, J. H. 1998, ApJ, 482, L167 